\title{
Influenza A Virus-Host Specificity: An Ongoing Cross-Talk Between Viral and Host Factors
}

\author{
Miaomiao Zhang ${ }^{1,2+}$, Mingbin Liu't, Shimeng Bai ${ }^{1}$, Chen Zhao', Zejun $\mathrm{Li}^{2 *}$, Jianqing $\mathrm{Xu}^{1 *}$ \\ and Xiaoyan Zhang ${ }^{1 *}$
}

'Scientific Research Center, Shanghai Public Health Clinical Center \& Institutes of Biomedical Sciences, Key Laboratory of Medical Molecular Virology of Ministry of Education/Health, Shanghai Medical College, Fudan University, Shanghai, China, ${ }^{2}$ Shanghai Veterinary Research Institute, Chinese Academic of Agricultural Sciences \& Animal Influenza Virus Evolution and Pathogenesis Innovation Team of the Agricultural Science and Technology Innovation Team, Shanghai, China

OPEN ACCESS

Edited by:

Chunfu Zheng,

University of Calgary, Canada

Reviewed by:

Zhenlong Liu,

McGill University, Canada

Yi-Quan Wu,

National Cancer Institute (NCl),

United States

${ }^{*}$ Correspondence:

Zejun Li

lizejun@shvri.ac.cn

Jianqing $X u$

xujianqing@fudan.edu.cn

Xiaoyan Zhang

zhangxiaoyan@fudan.edu.cn

tThese authors have contributed equally to this work

Specialty section:

This article was submitted to

Virology,

a section of the journal

Frontiers in Microbiology

Received: 16 September 2021

Accepted: 19 October 2021

Published: 05 November 2021

Citation:

Zhang M, Liu M, Bai S, Zhao C, Li Z,

$X u J$ and Zhang $X$ (2021)

Influenza A Virus-Host Specificity: An

Ongoing Cross-Talk Between Viral

and Host Factors.

Front. Microbiol. 12:777885.

doi: 10.3389/fmicb.2021.777885
One big threat from influenza A viruses (IAVs) is that novel viruses emerge from mutation alongside reassortment. Some of them have gained the capability to transmit into human from the avian reservoir. Understanding the molecular events and the involved factors in breaking the cross-species barrier holds important implication for the surveillance and prevention of potential influenza outbreaks. In this review, we summarize recent progresses, including several ground-breaking findings, in how the interaction between host and viral factors, exemplified by the PB2 subunit of the influenza virus RNA polymerase co-opting host ANP32 protein to facilitate transcription and replication of the viral genome, shapes the evolution of IAVs from host specificity to cross-species infection.

Keywords: influenza A virus, host specificity, pathogenesis, host factors, PB2

\section{INTRODUCTION}

Influenza A virus (IAV) is a major pathogen that greatly impacts human health and the poultry industry with a broad host tropism, capable of infecting various hosts, including wildfowl, swine, bat, and humans being. IAV is a negative-stranded RNA virus comprising eight segments. Two major surface proteins of IAV are hemagglutinin (HA) and neuraminidase (NA), which are used for classifying IAV subtypes based on sequence similarity. Up to date, $18 \mathrm{HA}$ and $11 \mathrm{NA}$ subtypes have been discovered, with H17, H18, N10, and N11 being only found in bats (Tong et al., 2013). Besides HA and NA, the IAV genome encodes at least 16 additional viral proteins, among which PB2, PB1, PA, HA, NP, NA, M1, M2, NS1, and NS2 proteins have been extensively characterized with relatively clear function while PB2-S1, PB1-F2, PB1-N40, PA-X, PA-N155, PA-N182, M42, and NS3 protein represent more recently discovered proteins (Vasin et al., 2014) and their functions remain largely unknown.

Birds are the major, if not the only, natural reservoir of IAV. Avian influenza viruses (AIV) can be divided into low-pathogenic (LPAIV) and highly pathogenic (HPAIV) strains, as determined by the presence of different types of the proteolytic cleavage site in HA (Horimoto and Kawaoka, 2005). The genetic diversity of IAVs is driven by an error-prone replication machinery, in addition to genetic reassortment events where viruses co-infecting the same cell exchange segments. As a result, a cross-species spillover can occasionally occur as an AIV mutant acquires the ability to transmit into aquatic (dolphins, seals, whales) or terrestrial 
mammals (pigs, horses, mink). Recent reports on human infection of AIV can be traced back to the year of 1997, when the contraction of H5N1 AIV killed 6 people in Hong Kong (Wong and Yuen, 2006). In 2003, an H7N7 AIV outbreak was reported in Netherlands, infecting 84 people with one death (Wong and Yuen, 2006). The threats of transmission of AIV to humans appear to continue growing: local outbreaks of highly pathogenic H5N1 AIV occurred in many Asia countries until 2004 (Stegeman et al., 2004); a total of 1,568 human infection H7N9 AIV has been reported, responsible for at least 617 deaths; the human spillover of H10N8 and H5N6 AIV were also observed (Chen et al., 2014; Yang et al., 2015). These indicate that, although breaking the cross-species barrier is difficult, there is always a likelihood of it happening with an expanding reservoir of AIV. Thus, exploring the molecular mechanisms underlying the host specificity of IAV and the events leading to their breach is an important step toward improving the preparedness of future influenza pandemics by instructing the development of enhanced surveillance of potential risk of avian-to-human transmission; identifying hidden virushost interfaces as new targets for antivirals. This review will summarize recent advances that start to unfold the complexity of species-specific virus-host interactions underpinning the host range of IAVs.

\section{VIRAL DETERMINANTS OF IAV HOST SPECIFICITY}

A clear announcement of host specificity of IAV is made because avian viruses generally show poor replication in mammalian cells, and vice versa. The host restriction of IAV has been linked to the difference between host of factors governing viral entry and multiple intracellular steps required for viral replication. Consequently, only AIVs with adaptive mutations, which account for a small portion of the virus pool that emerged from the natural reservoir, gain the ability to infect mammalian cells. These mutations are discussed in details per involved viral proteins.

\section{The Viral HA Protein}

The entry of IAV into host cells, representing the first species barrier to overcome, is mediated by viral surface hemagglutinin (HA) glycoprotein. Although all HA proteins have a general cell receptor in sialic acids (SA), a diverse family of sugar units terminally attached to glycans decorating surface glycoproteins and glycolipids, they show an origin-dependent preference for sialic acid-galactose linkage. HAs from human influenza viruses preferably recognize $\alpha-2,6$ linked SA ( $\alpha-2,6$ receptor) while those of avian origin showed a higher binding affinity with $\alpha$-2,3-linked SA ( $\alpha-2,3$ receptor; Matrosovich et al., $1997,2000)$. The correlation of this recognition pattern with SA distribution in human tissues explains why avian IAV normally cannot infect humans. In humans, $\alpha-2,6$ receptors are mainly expressed on cells of the ciliated epithelium along the upper respiratory tract (URT), while $\alpha-2,3$ receptors are primarily found in the lower respiratory tract (LRT; Shinya et al., 2006). In contrast, pig trachea presents both $\alpha-2,3$ and $\alpha-2,6$ receptors and is thus susceptible to infection of both human and avian virus, making pig a mixing vessel where new viruses can be generated through reassortment from parental virus of different origins.

Extensive structural studies have revealed the modes of interactions between HA and their corresponding SA receptor (Long et al., 2019). A wider receptor binding site (RBS) has been posited to form the basis of human-adapted HA accommodating $\alpha-2,6$ SA linkage, which appears bulkier than the $\alpha-2,3$ SA linkage. Several adaptive mutations in RBS of avian HAs have been shown to cause a switch of binding specificity, exemplified by E190D/G225D and A138S in H1 HA and Q226L/G228S in the HAs of H2 and H3 subtypes (Shi et al., 2014). These mutations increase binding affinity to the human receptor while reducing the binding to the avian receptor. The HA proteins of recently emerged H7N9 AIV that showed high pathogenicity in human infections appear to acquire some ability to bind to human receptor despite maintaining the preference for the avian receptor (Xu et al., 2013). This change was particularly exhibited by the Anhui-H7N9 virus, which harbors four mutations in RBS, namely S138A, G186V, T221P, and Q226L, accounting for the shift of receptor binding preference (Shi et al., 2013). As for the H5N1 virus, those causing human infections retain preference for avian receptor but such specificity can be skewed to human side by HA mutations in position 226 and 228, which are also required for airborne transmissibility (Stevens et al., 2008). Overall, typical AIV strains have not yet shown acquisition of transmissibility among humans until now. Despite the odds, the possibility of AIV breaking such a barrier cannot be ruled out, especially considering that multiple types of HA can attain airborne transmission ability in mammalian model systems (Kuchipudi et al., 2021).

\section{The Viral NA Protein}

As another major viral surface protein alongside HA, NA possesses sialidase activity and is responsible for cleaving SA from the cell surface to facilitate the release of the progeny virus particle (Schrauwen et al., 2014). Strong evidence supporting the adaptation of NA to host came from the short-stalk NA favored by poultry IAV, which is speculated to match short length of glycan linked to SA receptor (Li et al., 2010). However, this type of NA does not support airborne transmission in ferrets, likely due to ineffective cleavage with longer glycan and the resultant virus clumping. Thus, it has been proposed that a functional balance between the SA binding of HA and NA sialidase activity is a critical element involved in the host fitness of IAV (Lakdawala et al., 2015). Interestingly, a second HA binding site was found on some NAs, contributing to their hemadsorption (Hd) activity. Such Hd site was originally thought to regulate the catalytic activity of NA positively, but the recent characterization of NA protein from the H7N9 virus indicated that its existence might allow enhanced receptor binding, particularly to human-like $\alpha 2,6$-linked sialic acid 
(Benton et al., 2017). It remains to be determined whether the $\mathrm{Hd}$ site contributes to the ability of the H7N9 virus to infect humans infection.

\section{The Viral Polymerase Proteins}

The transcription and replication of the viral genome, carried out by the viral RNA polymerase, are central to viral growth and consequently become a critical barrier to overcome during the adaptation of IAV to a new host. The tripartite RNA-dependent RNA polymerase (RdRp) of IAV consists of polymerase protein basic 1 (PB1), polymerase protein basic 2 (PB2), and polymerase acidic protein (PA). Inside the virus, all the eight viral RNA (vRNA) segments are coated by nucleoprotein (NP) with RdRp binding to the conserved $5^{\prime}$ and $3^{\prime}$ ends to form viral ribonucleoprotein (vRNP) complexes (Nilsson et al., 2017). Once transported into the nucleus in infected cells, RdRp first transcribes vRNA into mRNA for viral protein production, a process initiated by a cap snatching mechanism wherein a nucleotide sequence of 10-20 nt length is cleaved from the $5^{\prime}$ end of host mRNAs by a sequential action of PB2 and PA and subsequently serve as the primer for viral mRNA synthesis (Dias et al., 2009; Yuan et al., 2009). The replication of the viral genome ensues after accumulation and nuclear transport of newly synthesized RdRp proteins. In contrast to transcription, the RdRp-mediated replication is primer independent and involves cRNA as an intermediate product. Additionally, NP is required for replication while dispensable for transcription. Recent studies also suggest that the catalytic activity of RdRp in vRNA replication requires dimer formation between an RNA-bound RdRp and free RdRp, which acts as a relay station to synthesize vRNA from cRNA (Nilsson et al., 2017). The central domain of RdRp is composed of PB1, the C-terminal domain of PA, the N-terminal one-third of PB2 encompassing the $\mathrm{N}$ terminus, the lid domain, and the N1 and N2 linkers (Nilsson et al., 2017).

The host adaptation of IAV RdRp is best displayed on PB2, which is responsible for cap-binding and participates in NP binding via regions in its $\mathrm{N}$ - and C-terminals. Several mutations in PB2 have been identified to be important for promoting adaptation of avian IAV to mammalian, epitomized by Glu-to-Lys substitution at position 627 (E627K) and Asp-to-Asn substitution at position 701 (D701N; Gabriel and Fodor, 2014). Subbarao et al. first reported the identity of amino acid at position 627 as a dividing line between avian and human influenza virus with respective $\mathrm{E}$ and $\mathrm{K}$ dominance (Subbarao et al., 1993). Importantly, a single change of $\mathrm{E}$ to $\mathrm{K}$ at position 627 was sufficient for restoring the ability of a PB2 single gene reassortant to replicate effectively in canine (MDCK) cells (Subbarao et al., 1993). Further analysis revealed that PB2 E627K mutation could remarkably raise the RdRp activity in mammalian but not avian cells, leading to an increased virulence in mammalian hosts' pathogenicity (Subbarao et al., 1993; Hatta et al., 2001; Gabriel et al., 2005). Indeed, $627 \mathrm{~K}$ is commonly shared by a wide spectrum of human pathogenic IAVs, including H5N1, H7N9, H10N8, and H5N6 subtypes (Gao et al., 2013; GarciaSastre and Schmolke, 2014; Yang et al., 2015). D701N as a mammalian-like signature was first identified by Li et al. through analyzing the genetic determinant underlying the ability of a duck H5N1 IAV to replicate in mice (Li et al., 2005). More recently, some $\mathrm{H} 9 \mathrm{~N} 2$ viruses isolated from minks were reported to contain the PB2 $701 \mathrm{~N}$ mutation and consequently showed enhanced virulence in mice compared to those without such mutation, supporting the potential of mink as a mixing vessel or intermediate host for H9N2 virus and possibly other IAVs (Xue et al., 2018).

Interestingly, other PB2 mutations, though less frequent than $627 \mathrm{~K}$ or $701 \mathrm{~N}$, can also promote human adaption. For example, the 2009 pandemic H1N1 virus harbors neither PB2 627K nor PB2 $701 \mathrm{~N}$ signatures (Mehle and Doudna, 2009). Instead, its mammalian adaptation was supported by PB2 G590S/G591R polymorphisms, which also conveyed enhanced RdRp activity in human cells (Mehle and Doudna, 2009). PB2-Q591K and K526R are two other mutations shown in H9N2 virus and H7N9/H5N1 viruses capable of conferring effective replication in mammalian cells in the absence of $627 \mathrm{~K}$ and $701 \mathrm{~N}$ (Song et al., 2014; Wang et al., 2016). The combinatorial effects of different adaptive mutations have also been studied. H7N9 virus bearing both $627 \mathrm{~K}$ and $526 \mathrm{R}$ replicates more efficiently in mammalian cells than those carrying the individual mutation, consistent with greater mortality in mice (Song et al., 2014). Mechanistically, the combination of 526R with $627 \mathrm{~K}$ appeared to optimize the interaction between PB2 and nuclear export protein (NEP), thereby promoting higher polymerase activity. A similar synergistic effect between PB2-526R and $701 \mathrm{~N}$ was also documented. In contrast, the co-existence of PB2 E627K and D701N mutations has not yet been detected in naturally occurring virus isolates, suggesting potential functional redundancy of the two mutations (Steel et al., 2009; Gabriel and Fodor, 2014).

Adaptive mutations of other components of IAV replication machinery were also identified, despite being less frequent than what was seen with PB2. A divergency at the position of PB1 protein of human influenza $\mathrm{A} / \mathrm{H} 1 \mathrm{~N} 1$ viruses was recently identified: This position was predominantly occupied by the avian-associated serine residue before switching to mammalianassociated glycine residue near the onset of the 2009 pandemic (Lin et al., 2019). Subsequent studies showed that PB1-216G was a lower RdRp fidelity variant than PB1-216S, thereby allowing the $\mathrm{H} 1 \mathrm{~N} 1$ virus to yield adaptive mutations at higher rates and promoting viral epidemiological fitness (Lin et al., 2019). Multiple studies revealed the role of PA in the human adaptation of AIV. It was found that, in avian H9N2 background, a single replacement of PA segment from 2009 pandemic H1N1 virus resulted in enhanced viral polymerase activity, leading to a higher pathogenicity in infected mice (Sun et al., 2011). A systematic comparison of a panel of reassortant viruses between a duck-derived $\mathrm{H} 5 \mathrm{~N} 1$ and a highly transmissible human-infective H1N1 virus showed that PA and nonstructural gene are the major $\mathrm{H} 1 \mathrm{~N} 1$ determinants of droplet-mediated transmission between guinea pigs (Zhang et al., 2013). A couple of human adaptive PA mutations were identified, including E349G and PA 97I, with a positive impact on viral polymerase activity in mammalian cells (Song et al., 2009). Evidence 
supports the concerted action between mutations of different RdRp subunits and NP protein in enhancing human adaptation. Gabriel et al. reported that mutations in PB2, PA, and NP, namely PB2 $701 \mathrm{~N}$ and $714 \mathrm{R}, \mathrm{PA} 615 \mathrm{~N}$, and NP 319K, cooperatively enhance AIV SC35 (H7N7) polymerase activity and consequently enable an effective replication in mammalian cells (Gabriel et al., 2005). The coupling between PB2 D701N and NP D319K mutation was confirmed in other studies (Gabriel et al., 2011). The molecular mechanism(s) underpinning synergy between these mutations in enhancing mammalian fitness of AIV has yet to be fully delineated, though some studies, as described below, suggested the involvement of importin- $\alpha$.

\section{NS1A and Other Viral Proteins}

The interferon (IFN)-centered innate immune response represents the first-line defense against viral infection. Accordingly, IAV develops several viral countermeasures epitomized by nonstructural protein 1 (NS1). NS1 protein is produced by the co-linear transcript of RNA segment 8, which also encodes NS2 (NEP) through alternative splicing (Liu et al., 1997). NS1A comprises an RNA binding domain (RBD) and an effector domain (ED); both domains contribute to the ability of NS1A to use multiple rather than single tactics to evade the IFN antiviral response (Yin et al., 2007; Bornholdt and Prasad, 2008). Two main mechanisms have been proposed for the IFN countering the action of NS1A, namely blocking the activation of RIG-I, the major pattern recognition receptor responsible for sensing the incoming IAV genome, and inhibiting the activity of CPSF30 (Cleavage and polyadenylation specificity factor $30 \mathrm{kDa}$ subunit) from suppressing the maturation and consequently the nuclear export of IFN mRNA (Cho et al., 2020; Rosário-Ferreira et al., 2020). The RNA-binding of NS1, once thought to sequester dsRNA produced by viral replication from host dsRNA sensor, was shown to primarily function as a competitor of $2^{\prime}-5^{\prime}$-oligoadenylate synthetase (OAS) to suppress the RNaseL antiviral pathway (Min and Krug, 2006). NS1A is also engaged in interactions with various host factors without known roles in innate immune response, implicating additional regulatory roles in other aspects of the viral cycle (RosárioFerreira et al., 2020). The wide spectrum of host protein interactions by NS1A has been proposed as a result of the ready-for-exploitation nature of its ED (Cho et al., 2020) and/ or a double-stranded RNA platform that strengthens the weak interactions NS1A with certain host factors (Chen et al., 2020).

Several naturally occurring mutations of NS1 have been linked to enhanced viral replication and pathogenicity, including D92E, P42S, and deletion of 80-84 residues (del80-84). As expected, these mutations were often associated with increased virus resistance to interferon (Seo et al., 2002). It is a bit of surprise that the CPSF30 binding site of NS1A is not conserved across IAVs: Although the majority of NS1A proteins bind to CPSF30, those from pandemic H1N1 2009 virus and humaninfective H5N1 1997 virus cannot (Long et al., 2019), which leads to a hypothesis that gain or loss of CPSF binding of NS1A may be a viral means to balance between viral replication and interferon antagonism on the road toward mammalian adaptation. Sequence analyses also pinpoint several amino changes that could associate with the adaptation of AIV to mammalian host, such as the alteration of last amino acids from ESEV to RSKV (Zielecki et al., 2010), and the D125G substitution introducing an extra splice site into the NS gene (Selman et al., 2012). However, whether and how these changes may influence the host range of AIV remained to be determined. Overall, a link between NS1 mutations and cross-species transmission of AIVs is still elusive.

Apart from NS1A, the human adaptation of AIV may be facilitated by mutating viral accessory proteins that were recently discovered, such as $\mathrm{PB} 1-\mathrm{F} 2$ and $\mathrm{PA}-\mathrm{X}$. $\mathrm{PB} 1-\mathrm{F} 2$ is an 87-amino acid small protein produced from $\mathrm{PB} 1$ encoding sequence through +1 frameshift and has been shown to antagonize antiviral innate immunity possibly via interaction with components of the RIG-I/MAVS system, particularly the mitochondrial antiviral signaling (MAVS) protein (Mazur et al., 2008). Interaction between PB1-F2 and PB1 has also been documented and is in line with the enhancing effect of PB1-F2 on the RdRp activity (Mazur et al., 2008). The role of PB1-F2 in the viral pathogenesis was highlighted by the finding that a single amino acid substitution in PB1-F2, namely N66S, is associated with increased virulence of the 1997 human H5N1 virus is also conserved in the 1918 pandemic virus (Conenello et al., 2007). However, the ORF of PB1-F2 is often truncated or lost in swine or human IAVs, raising the speculation that PB1-F2 is not essential for IAV mammalian adaptation or a compensatory mechanism has been acquired (Long et al., 2019).

PA-X is the product of +1 ribosomal frameshifting during translation of PA protein, retaining the 191-aa long $\mathrm{N}$-terminal endonuclease domain while acquiring a unique C-terminal region known as X-ORF, commonly encoding 61 aa (Jagger et al., 2012). PA-X is known to facilitate viral growth by coordinating the virus-mediated shutoff of host gene expression (Gaucherand et al., 2019). Interestingly, some IAV strains possess early stop codon in the X-ORF, resulting in a truncated PA-X protein 41 aa in length (Sun et al., 2020). Avian, equine, and human seasonal H3N2 and H1N1 influenza viruses generally encode full-length $\mathrm{X}-\mathrm{ORF}$ while truncated $\mathrm{X}$-ORF is characteristic of the 2009 pandemic H1N1, swine and canine viruses (Sun et al., 2020). In terms of swine influenza viruses (SIVs), the majority of early isolates were found to express a full-length PA-X until 1985, from when isolates featuring truncated PA-X gradually increased to become the dominant species, implicating a selection favoring truncated PA-X in pigs (Xu et al., 2016). Indeed, $\mathrm{Xu}$ et al. demonstrated that, in the background of a "triple-reassortment" H1N2 SIV, mutating X-ORF to express a full-length PA-X resulted in attenuated viral replication and transmission in pigs (Xu et al., 2017). By contrast, an investigation based on the 2009 pandemic H1N1 revealed that full-length PA-X protein supported increased virulence in mice relative to the truncated form (Gao et al., 2015). Thus, PA-X is a likely contributing factor to the host specificity of IAV. The notion was further strengthened by the finding that single human-specific amino acid substitutions in PA-X, particularly the R195K mutation, might underpin the animal-to-human jump of H7N9, H5N6, and H1N1/2009 viruses (Sun et al., 2020). 


\section{INTRACELLULAR HOST FACTORS UNDERLYING THE HOST SPECIFICITY OF IAV: IMPORTIN- $\alpha$ AND ANP32A TAKE THE LEAD}

A major recent advance in understanding the host specificity of IAV is identifying the related intracellular host factors, best represented by importin- $\alpha$ and ANP32 proteins.

\section{Importin- $\alpha$}

In uninfected cells, importin- $\alpha$ functions as an adaptor protein involved in the nuclear transportation of host proteins possessing nuclear localization signal (NLS; Stewart, 2007). IAV-infected cells are usurped by the virus to transport incoming vRNPs into the nucleus and are also responsible for nuclear importation of newly synthesized PB2 and NP proteins following primary transcription, which is a requisite for sustained viral replication (Stewart, 2007). Avian and human IAVs appear to engage different importin- $\alpha$ isoforms for effective viral replication as revealed using importin- $\alpha$-silenced cells and importin- $\alpha$-knockout mice: Avian virus depends on importin- $\alpha 3$, whereas human virus displays a switch to importin- $\alpha 7$ dependency; the 2009 H1N1 pandemic IAV instead showed a dual dependency of both importin- $\alpha 3$ and importin- $\alpha$, suggesting ongoing viral adaptation (Gabriel et al., 2011; Pumroy et al., 2015). Consistent with the viral replication data, avian- and human-like PB2 were found to bind preferentially to importin- $\alpha 3$ and importin- $\alpha 7$, respectively (Gabriel et al., 2011). Further evidence that differential importin- $\alpha$ recognition is a mechanism of IAV host specificity came from binding studies that D701N mutation enhances the interaction between PB2 and importin- $\alpha 7$, so does D319K for NP (Gabriel et al., 2011). Given the proximity of 701 to the NLS of PB2, it has been speculated that the D701N mutation might induce a conformation change that facilitates a stronger binding to importin- $\alpha$ (Tarendeau et al., 2007; Sediri et al., 2015). Interestingly, a most recent study identified a correlation between PB2-D701N/NP-D319K mutations and reduced expression of importin- $\alpha 3$ in the respiratory tract of IAV-infected humans and mice. As importin- $\alpha 3$ mediates the nuclear import of NF- $\kappa b$ and is thus required for induction of various antiviral genes in IAV-infected cells, its downregulation complements the virus's capability to access importin- $\alpha 7$ in ensuring that virus can gain replicative fitness in the human lung (Gabriel et al., 2008; Thiele et al., 2020).

\section{ANP32 Proteins}

Once in the nucleus, the IAV viral polymerase needs to utilize host machinery for effective transcription and genome replication, and this represents another major barrier that AIV needs to surpass upon avian-mammalian adaptation. The original clue for this barrier came from the observation that the poor activity of avian IAV polymerase in mammalian cells could be rescued by fusion with avian cells to form heterokaryons, which can be explained by the absence of a supportive avian host factor in mammalian cells. Long et al. first discovered this longed-for host factor as chicken ANP32A. Surprisingly, there is a human homolog of chicken ANP32A (chANP32A); the major difference between chicken and human ANP32A is that the former possesses an additional 33 amino acids between the shared leucine-rich repeats and carboxy-terminal low-complexity acidic region domains owing to exon duplication (Long et al., 2016; Figure 1). Importantly, the 33-aa insertion was responsible for the superiority of chicken ANP32A over human ANP32A in supporting the activity of avian PB2-627E viral polymerase in mammalian cells (Long et al., 2016; Domingues and Hale, 2017). In this regard, the PB2 E627K mutation was selected to meet the viral need to co-opt the mammalian ANP32A for effective viral replication.

Subsequent studies further revealed deep connections between ANP32 family proteins and human adaption of IAV polymerase. The activity of ANP32A appears to be regulated by alternative splicing in avian cells, resulting in variants containing essential no (human-like), partial, or full 33aa-insertion (Baker et al., 2018). More interestingly, the usage of ANP32A splicing sites varies across different bird species, and some species predominantly express human-like ANP32A, most pronounced in swan goose (>70\%) and ostrich (close to $100 \%$; Domingues et al., 2019). It is thus proposed that the differential ANP32A splicing inherent to natural avian hosts may provide a pre-adaptation mechanism that shapes the evolution of PB2 to facilitate cross-species transmission (Domingues et al., 2019). A recent finding further strengthened the view of ANP32A as a facilitator of IAV evolution. Swine ANP32A is capable

A

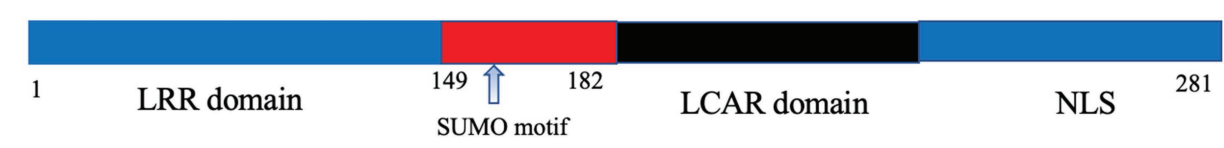

B

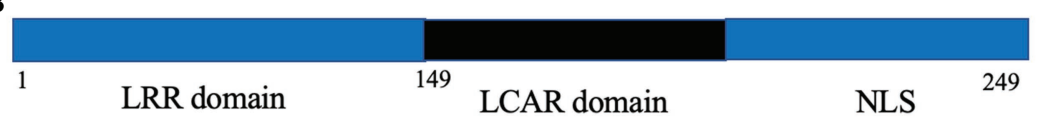

FIGURE 1 | Schematic illustration of chicken (A) and human ANP32A (B). The avian-specific 33-amino acid insertion is highlighted in red with the containing SUMO binding motif being indicated by an arrow. 
of supporting avian-type IAV polymerase activity, although to a less extent as compared with avian ANP32A, consistent with the pig as an important intermediate host for zoonotic IAVs (Zhang et al., 2020a). This unique feature of swine ANP32A was attributed to $106 \mathrm{~V}$, which exhibits positive epistasis with $156 \mathrm{~S}$ and has not been found in other studied vertebrate species (Zhang et al., 2020a). Recent studies also revealed another member of ANP32 family, ANP32B, in regulating IAV polymerase activity. This revelation was made using human ANP32A and ANP32B single- and double-knockout cells as only in the latter cells was the viral polymerase activity abrogated (Zhang et al., 2019). The analysis also indicated that human ANP32B is a more potent pro-viral factor than human ANP32A. In contrast, the viral polymerase supporting activity was lost in chicken ANP32B, and further dissection pinpointed residue 129 and 130 as the critical determinant of ANP32B activity: Avian ANP32B possesses isoleucine and asparagine at the two positions, whereas in human ANP32A and ANP32B, as well as avian ANP32A, they are filled with asparagine and aspartate (Zhang et al., 2019).

The precise mechanism by which ANP32 regulates the activity of IAV RNA polymerase remains unclear. ANP32A or ANP32B was associated with the trimeric viral polymerase in infected cells or when they were simultaneously expressed by co-transfection (Zhang et al., 2019). The failure of ANP32A to bind to free PB2 in co-expressed cells indicated that such association requires the formation of a viral polymerase complex. On the other hand, in vitro binding assay employing purified protein revealed a direct interaction between ANP32A and the 627 domain of PB2. However, the binding of human or chicken ANP32A to viral polymerase appears to be independent of PB2-627 identity (Domingues and Hale, 2017). Following the finding that chicken ANP32A binds to PB2 stronger than human ANP32A, Domingues et al. discovered a sumo interaction motif (SIM) in the first four residues of the avian-specific 33-aa insertion and suggested it might account for increased binding of chicken ANP32A to PB2 (Domingues and Hale, 2017). However, a natural splicing variant of chicken ANP32A with SIM deletion can support viral polymerase activity, though less effective than that with a complete 33-aa insertion (Domingues and Hale, 2017). Recently, Baker et al. provided experimental evidences linking ANP32A to a specific form of the viral polymerase (Baker et al., 2018). The binding of ANP32A to viral polymerase in co-transfected cells was enhanced in the presence of viral genomic RNA. It was known that the low activity of PB2 $627 \mathrm{E}$ viral polymerase can be trans-complemented by mutations at positions 3 and 5 of $3^{\prime}$ vRNA promoter. When assayed on reporter bearing such mutant vRNA promoter, chANP32A showed a little enhancing effect, implying the same or similar mechanism shared by a mutant promoter and chANP32A (Baker et al., 2018). These results are consistent with the hypothesis that ANP32A facilitates the assembly of either cRNP, vRNP, or both.

Structural approaches have also searched the molecular basis for recognition of ANP32A by PB2. Using a combination of NMR and quantitative ensemble analysis, Camacho-Zarco et al. conducted a comparative analysis of complex formed between human ANP32A and human-adapted PB2-627-NLS domain (K627 form) versus that comprising avian ANP32A and avian adapted PB2-627-NLS domain (Camacho-Zarco et al., 2020). Although both complexes were highly dynamic, they were found to display two different modes of interaction: Human ANP32A utilizes a track of positively charged residues including K627 to maximize interaction with highly acidic IDD domain (alternative name of LCAR to highlight its low complexity); with such positive charged surface being interrupted by E627, avian ANP32A can compensate this loss by broadening the sampling of IDD domain to exploit more expanded interaction surface, particularly involving a hexapeptide motif present in the avian-specific 33-aa insertion (Camacho-Zarco et al., 2020). Despite providing the first structural insight into host-specific ANP32A-PB2 interaction, the study from Camacho-Zarco et al. could not interpret the previous observation that chicken ANP32A binds to PB2 more strongly than its human ortholog. Instead, it would predict a higher binding affinity between human ANP32A and 627K type PB2. More recently, Carrique et al. reported cryo-EM structures of influenza $\mathrm{C}$ virus polymerase (FluPolC) in complex with human or avian ANP32A (Carrique et al., 2020). In both structures, two FluPolC molecules, one bound to a 47-nt long vRNA and one staying free, formed an asymmetric dimer that ANP32A bridges through its LRR domain (Carrique et al., 2020). The LCAR domain of ANP32A further stabilizes the complex formation in the FluPolC-chicken ANP32A structure with the avian-specific 33-aa insertion directly interacting with the two juxtaposed 627 domains of PB2 (Carrique et al., 2020). The authors thus proposed that ANP32A-FluPol dimer functions as a platform for viral genome replication, where a vRNA-bound $\mathrm{FluPol}_{\mathrm{R}}$ and a free $\mathrm{FluPol}_{\mathrm{B}}$ act sequentially in cRNA synthesis and the ensued vRNP assembly (Carrique et al., 2020). However, chicken ANP32A, unlike human ANP32A/B, has limited ability to support the viral polymerase of the influenza B virus (Zhang et al., 2020b), whether such platform is shared by IAV viral polymerase remains to be determined.

\section{CONCLUSION}

The host specificity of the IAV is underpinned by complex interactions between viral proteins and host factors. This complexity is translated to the requirement of a constellation of mutations for the virus to adapt to a new host. Although recent studies begin to reveal the molecular basis of several human adaptive mutations, exemplified by PB2 627K, many questions remain to be addressed. For example, no clear consensus has been reached regarding the exact mechanism by which ANP32A is co-opted to enhance viral polymerase activity in a species-specific manner. To answer this, we might need a structure of ANP32A in complex with the matched viral polymerase of IAV. It is also unclear when the human adaptive mutations are selected in the human cells after transmission or preexisted in the animal side. In the case of 
PB2 627K, the presence of human-like ANP32A in some bird species and the ability of swine ANP32B to support a certain degree of PB2 $627 \mathrm{~K}$ viral polymerase activity would suggest the second possibility. Identifying PB1-216G, which lowers the viral polymerase activity, reminds us that changing the intrinsic property of viral polymerase may lead to adaptive mutations.

Developing an effective countermeasure against innate immunity of new host is another essential stepstone for AIV to cross the species barrier. It is recognized that the outstanding ability of chicken cells to support AIV growth is at least partially ascribed to a compromised type I IFN system, i.e., lacking of a functional RIG-I homolog (Barber et al., 2010). In addition, the chicken version of Mx protein, which is a major IFN-induced anti-influenza effector molecule in mammal setting, is not capable of restricting influenza virus replication (Benfield et al., 2008). NS1A is the most likely viral candidate taking the assignment to cope with the challenge of more powerful innate immunity in mammal cells. Elucidating the relationship between the evolution of NS1A and adaptation of AIV to mammalian hosts and the underlying mechanism warrant future investigation.

It should also be noticed that environmental and physiological factors, which we have not discussed here, also play an important role in the host restriction of IAV, particularly the transmissibility, which adds more constraints to inter-species transmission. A critical hidden constraint is recently revealed by an elegant study of Soh et al., demonstrating that the architecture of genome code makes some potential human adaption mutations

\section{REFERENCES}

Baker, S. F., Ledwith, M. P., and Mehle, A. (2018). Differential splicing of ANP32A in birds alters its ability to stimulate RNA synthesis by restricted influenza polymerase. Cell Rep. 24, 2581.e4-2588.e4. doi: 10.1016/j. celrep.2018.08.012

Barber, M. R., Aldridge, J. R. Jr., Webster, R. G., and Magor, K. E. (2010). Association of RIG-I with innate immunity of ducks to influenza. Proc. Natl. Acad. Sci. U. S. A. 107, 5913-5918. doi: 10.1073/pnas.1001755107

Benfield, C. T., Lyall, J. W., Kochs, G., and Tiley, L. S. (2008). Asparagine 631 variants of the chicken Mx protein do not inhibit influenza virus replication in primary chicken embryo fibroblasts or in vitro surrogate assays. J. Virol. 82, 7533-7539. doi: 10.1128/JVI.00185-08

Benton, D. J., Wharton, S. A., Martin, S. R., and McCauley, J. W. (2017). Role of neuraminidase in influenza A (H7N9) virus receptor binding. J. Virol. 91:e02293-16. doi: 10.1128/JVI.02293-16

Bornholdt, Z. A., and Prasad, B. V. (2008). X-ray structure of NS1 from a highly pathogenic H5N1 influenza virus. Nature 456, 985-988. doi: 10.1038/ nature 07444

Camacho-Zarco, A. R., Kalayil, S., Maurin, D., Salvi, N., Delaforge, E., Milles, S., et al. (2020). Molecular basis of host-adaptation interactions between influenza virus polymerase PB2 subunit and ANP32A. Nat. Commun. 11:3656. doi: 10.1038/s41467-020-17407-x

Carrique, L., Fan, H., Walker, A. P., Keown, J. R., Sharps, J., Staller, E., et al. (2020). Host ANP32A mediates the assembly of the influenza virus replicase. Nature 587, 638-643. doi: 10.1038/s41586-020-2927-z

Chen, G., Ma, L. C., Wang, S., Woltz, R. L., Grasso, E. M., Montelione, G. T., et al. (2020). A double-stranded RNA platform is required for the interaction between a host restriction factor and the NS1 protein of influenza A virus. Nucleic Acids Res. 48, 304-315. doi: 10.1093/nar/gkz1094

Chen, H., Yuan, H., Gao, R., Zhang, J., Wang, D., Xiong, Y., et al. (2014). Clinical and epidemiological characteristics of a fatal case of avian influenza impossible because they are inaccessible to single nucleotide substitution (Soh et al., 2019). Extension of the comprehensive, active search for potential human adaption mutations to other viral proteins might provide us a map of risk spots on viral proteins that we need to be aware of when evaluating the cross-species transmission potential of avian viruses. Finally, further exploration of mechanisms underlying the host specificity and cross-species infection is expected to help dissect the complicated network of host factors usurped by IAVs, thus providing new targets for developing therapeutics and antiviral drugs.

\section{AUTHOR CONTRIBUTIONS}

$\mathrm{XZ}$, JX, and ZL contributed to conception and design of the study. MZ, ML, and SB organized the database and performed the statistical analysis. MZ and CZ wrote the manuscript. All authors contributed to manuscript revision and approved the submitted version.

\section{FUNDING}

This work was supported by the National Natural Science Foundation of China (81771704, 82071788, 31902276), the Shanghai Pujiang Program (19PJ1409100), and the intramural Funding from Shanghai Public Health Clinical Center (to MZ).

A H10N8 virus infection: a descriptive study. Lancet 383, 714-721. doi: 10.1016/S0140-6736(14)60111-2

Cho, J. H., Zhao, B., Shi, J., Savage, N., Shen, Q., Byrnes, J., et al. (2020). Molecular recognition of a host protein by NS1 of pandemic and seasonal influenza A viruses. Proc. Natl. Acad. Sci. U. S. A. 117, 6550-6558. doi: $10.1073 /$ pnas. 1920582117

Conenello, G. M., Zamarin, D., Perrone, L. A., Tumpey, T., and Palese, P. (2007). A single mutation in the PB1-F2 of H5N1 (HK/97) and 1918 influenza A viruses contributes to increased virulence. PLoS Pathog. 3, 1414-1421. doi: 10.1371/journal.ppat.0030141

Dias, A., Bouvier, D., Crepin, T., McCarthy, A. A., Hart, D. J., Baudin, F., et al. (2009). The cap-snatching endonuclease of influenza virus polymerase resides in the PA subunit. Nature 458, 914-918. doi: 10.1038/nature07745

Domingues, P., Eletto, D., Magnus, C., Turkington, H. L., Schmutz, S., Zagordi, O., et al. (2019). Profiling host ANP32A splicing landscapes to predict influenza A virus polymerase adaptation. Nat. Commun. 10:3396. doi: 10.1038/ s41467-019-11388-2

Domingues, P., and Hale, B. G. (2017). Functional insights into ANP32Adependent influenza A virus polymerase host restriction. Cell Rep. 20, 2538-2546. doi: 10.1016/j.celrep.2017.08.061

Gabriel, G., Dauber, B., Wolff, T., Planz, O., Klenk, H. D., and Stech, J. (2005). The viral polymerase mediates adaptation of an avian influenza virus to a mammalian host. Proc. Natl. Acad. Sci. U. S. A. 102, 18590-18595. doi: 10.1073/pnas.0507415102

Gabriel, G., and Fodor, E. (2014). Molecular determinants of pathogenicity in the polymerase complex. Curr. Top. Microbiol. Immunol. 385, 35-60. doi: 10.1007/82_2014_386

Gabriel, G., Herwig, A., and Klenk, H. D. (2008). Interaction of polymerase subunit $\mathrm{PB} 2$ and NP with importin alpha1 is a determinant of host range of influenza A virus. PLoS Pathog. 4:e11. doi: 10.1371/journal.ppat.0040011

Gabriel, G., Klingel, K., Otte, A., Thiele, S., Hudjetz, B., Arman-Kalcek, G., et al. (2011). Differential use of importin- $\alpha$ isoforms governs cell tropism 
and host adaptation of influenza virus. Nat. Commun. 2:156. doi: 10.1038/ ncomms 1158

Gao, R. B., Cao, B., Hu, Y. W., Feng, Z. J., Wang, D. Y., Hu, W. F., et al. (2013). Human infection with a novel avian-origin influenza A (H7N9) virus. N. Engl. J. Med. 368, 1888-1897. doi: 10.1056/NEJMoa1304459

Gao, H., Sun, H., Hu, J., Qi, L., Wang, J., Xiong, X., et al. (2015). Twenty amino acids at the $\mathrm{C}$-terminus of PA-X are associated with increased influenza A virus replication and pathogenicity. J. Gen. Virol. 96, 2036-2049. doi: 10.1099/vir.0.000143

Garcia-Sastre, A., and Schmolke, M. (2014). Avian influenza A H10N8-a virus on the verge? Lancet 383, 676-677. doi: 10.1016/S0140-6736(14)60163-X

Gaucherand, L., Porter, B. K., Levene, R. E., Price, E. L., Schmaling, S. K., Rycroft, C. H., et al. (2019). The influenza A virus endoribonuclease PA-X usurps host mRNA processing machinery to limit host gene expression. Cell Rep. 27, 776.e7-792.e7. doi: 10.1016/j.celrep.2019.03.063

Hatta, M., Gao, P., Halfmann, P., and Kawaoka, Y. (2001). Molecular basis for high virulence of Hong Kong H5N1 influenza A viruses. Science 293, 1840-1842. doi: 10.1126/science. 1062882

Horimoto, T., and Kawaoka, Y. (2005). Influenza: lessons from past pandemics, warnings from current incidents. Nat. Rev. Microbiol. 3, 591-600. doi: 10.1038/ nrmicro1208

Jagger, B. W., Wise, H. M., Kash, J. C., Walters, K. A., Wills, N. M., Xiao, Y. L., et al. (2012). An overlapping protein-coding region in influenza A virus segment 3 modulates the host response. Science 337, 199-204. doi: 10.1126/ science. 1222213

Kuchipudi, S. V., Nelli, R. K., Gontu, A., Satyakumar, R., Surendran Nair, M., and Subbiah, M. (2021). Sialic acid receptors: the key to solving the enigma of zoonotic virus spillover. Viruses 13:262. doi: 10.3390/v13020262

Lakdawala, S. S., Jayaraman, A., Halpin, R. A., Lamirande, E. W., Shih, A. R., Stockwell, T. B., et al. (2015). The soft palate is an important site of adaptation for transmissible influenza viruses. Nature 526, 122-125. doi: 10.1038/nature 15379

Li, Z., Chen, H., Jiao, P., Deng, G., Tian, G., Li, Y., et al. (2005). Molecular basis of replication of duck $\mathrm{H} 5 \mathrm{~N} 1$ influenza viruses in a mammalian mouse model. J. Virol. 79, 12058-12064. doi: 10.1128/JVI.79.18.12058-12064.2005

Li, Q., Qi, J., Zhang, W., Vavricka, C. J., Shi, Y., Wei, J., et al. (2010). The 2009 pandemic H1N1 neuraminidase N1 lacks the 150-cavity in its active site. Nat. Struct. Mol. Biol. 17, 1266-1268. doi: 10.1038/nsmb.1909

Lin, R. W., Chen, G. W., Sung, H. H., Lin, R. J., Yen, L. C., Tseng, Y. L., et al. (2019). Naturally occurring mutations in PB1 affect influenza A virus replication fidelity, virulence, and adaptability. J. Biomed. Sci. 26:55. doi: 10.1186/s12929-019-0547-4

Liu, J., Lynch, P. A., Chien, C. Y., Montelione, G. T., Krug, R. M., and Berman, H. M. (1997). Crystal structure of the unique RNA-binding domain of the influenza virus NS1 protein. Nat. Struct. Biol. 4, 896-899. doi: 10.1038/ nsb1197-896

Long, J. S., Giotis, E. S., Moncorge, O., Frise, R., Mistry, B., James, J., et al. (2016). Species difference in ANP32A underlies influenza A virus polymerase host restriction. Nature 529, 101-104. doi: 10.1038/nature16474

Long, J. S., Mistry, B., Haslam, S. M., and Barclay, W. S. (2019). Host and viral determinants of influenza A virus species specificity. Nat. Rev. Microbiol. 17, 67-81. doi: 10.1038/s41579-018-0115-Z

Matrosovich, M. N., Gambaryan, A. S., Teneberg, S., Piskarev, V. E., Yamnikova, S. S., Lvov, D. K., et al. (1997). Avian influenza A viruses differ from human viruses by recognition of sialyloligosaccharides and gangliosides and by a higher conservation of the HA receptor-binding site. Virology 233, 224-234. doi: 10.1006/viro.1997.8580

Matrosovich, M., Tuzikov, A., Bovin, N., Gambaryan, A., Klimov, A., Castrucci, M. R., et al. (2000). Early alterations of the receptor-binding properties of $\mathrm{H} 1, \mathrm{H} 2$, and $\mathrm{H} 3$ avian influenza virus hemagglutinins after their introduction into mammals. J. Virol. 74, 8502-8512. doi: 10.1128/ JVI.74.18.8502-8512.2000

Mazur, I., Anhlan, D., Mitzner, D., Wixler, L., Schubert, U., and Ludwig, S. (2008). The proapoptotic influenza A virus protein PB1-F2 regulates viral polymerase activity by interaction with the PB1 protein. Cell. Microbiol. 10, 1140-1152. doi: 10.1111/j.1462-5822.2008.01116.x

Mehle, A., and Doudna, J. A. (2009). Adaptive strategies of the influenza virus polymerase for replication in humans. Proc. Natl. Acad. Sci. U. S. A. 106, 21312-21316. doi: 10.1073/pnas.0911915106
Min, J. Y., and Krug, R. M. (2006). The primary function of RNA binding by the influenza A virus NS1 protein in infected cells: inhibiting the $2^{\prime}-5^{\prime}$ oligo (A) synthetase/RNase L pathway. Proc. Natl. Acad. Sci. U. S. A. 103, 7100-7105. doi: 10.1073/pnas.0602184103

Nilsson, B. E., Te Velthuis, A. J. W., and Fodor, E. (2017). Role of the PB2 627 domain in influenza A virus polymerase function. J. Virol. 91:e02467-16. doi: 10.1128/JVI.02467-16

Pumroy, R. A., Ke, S., Hart, D. J., Zachariae, U., and Cingolani, G. (2015). Molecular determinants for nuclear import of influenza A PB2 by importin $\alpha$ isoforms 3 and 7. Structure 23, 374-384. doi: 10.1016/j.str.2014.11.015

Rosário-Ferreira, N., Preto, A. J., Melo, R., Moreira, I. S., and Brito, R. M. M. (2020). The central role of non-structural protein 1 (NS1) in influenza biology and infection. Int. J. Mol. Sci. 21:1511. doi: 10.3390/ijms21041511

Schrauwen, E. J., de Graaf, M., Herfst, S., Rimmelzwaan, G. F., Osterhaus, A. D., and Fouchier, R. A. (2014). Determinants of virulence of influenza A virus. Eur. J. Clin. Microbiol. Infect. Dis. 33, 479-490. doi: 10.1007/s10096-013-1984-8

Sediri, H., Schwalm, F., Gabriel, G., and Klenk, H. D. (2015). Adaptive mutation PB2 D701N promotes nuclear import of influenza vRNPs in mammalian cells. Eur. J. Cell Biol. 94, 368-374. doi: 10.1016/j.ejcb.2015.05.012

Selman, M., Dankar, S. K., Forbes, N. E., Jia, J. J., and Brown, E. G. (2012). Adaptive mutation in influenza A virus non-structural gene is linked to host switching and induces a novel protein by alternative splicing. Emerg. Microbes Infect. 1:e42. doi: 10.1038/emi.2012.38

Seo, S. H., Hoffmann, E., and Webster, R. G. (2002). Lethal H5N1 influenza viruses escape host anti-viral cytokine responses. Nat. Med. 8, 950-954. doi: $10.1038 / \mathrm{nm} 757$

Shi, Y., Wu, Y., Zhang, W., Qi, J. X., and Gao, G. F. (2014). Enabling the 'host jump': structural determinants of receptor-binding specificity in influenza A viruses. Nat. Rev. Microbiol. 12, 822-831. doi: 10.1038/nrmicro3362

Shi, Y., Zhang, W., Wang, F., Qi, J., Wu, Y., Song, H., et al. (2013). Structures and receptor binding of hemagglutinins from human-infecting H7N9 influenza viruses. Science 342, 243-247. doi: 10.1126/science.1242917

Shinya, K., Ebina, M., Yamada, S., Ono, M., Kasai, N., and Kawaoka, Y. (2006). Avian flu: influenza virus receptors in the human airway. Nature 440, 435-436. doi: $10.1038 / 440435 \mathrm{a}$

Soh, Y. S., Moncla, L. H., Eguia, R., Bedford, T., and Bloom, J. D. (2019). Comprehensive mapping of adaptation of the avian influenza polymerase protein PB2 to humans. eLife 8:e45079. doi: 10.7554/eLife.45079

Song, M. S., Pascua, P. N., Lee, J. H., Baek, Y. H., Lee, O. J., Kim, C. J., et al. (2009). The polymerase acidic protein gene of influenza A virus contributes to pathogenicity in a mouse model. J. Virol. 83, 12325-12335. doi: 10.1128/ JVI.01373-09

Song, W., Wang, P., Mok, B. W., Lau, S. Y., Huang, X., Wu, W. L., et al. (2014). The K526R substitution in viral protein PB2 enhances the effects of E627K on influenza virus replication. Nat. Commun. 5:5509. doi: 10.1038/ ncomms6509

Steel, J., Lowen, A. C., Mubareka, S., and Palese, P. (2009). Transmission of influenza virus in a mammalian host is increased by PB2 amino acids 627K or 627E/701N. PLoS Pathog. 5:e1000252. doi: 10.1371/journal. ppat. 1000252

Stegeman, A., Bouma, A., Elbers, A. R., de Jong, M. C., Nodelijk, G., de Klerk, F., et al. (2004). Avian influenza A virus (H7N7) epidemic in The Netherlands in 2003: course of the epidemic and effectiveness of control measures. J. Infect. Dis. 190, 2088-2095. doi: 10.1086/425583

Stevens, J., Blixt, O., Chen, L. M., Donis, R. O., Paulson, J. C., and Wilson, I. A. (2008). Recent avian H5N1 viruses exhibit increased propensity for acquiring human receptor specificity. J. Mol. Biol. 381, 1382-1394. doi: 10.1016/j. jmb.2008.04.016

Stewart, M. (2007). Molecular mechanism of the nuclear protein import cycle. Nat. Rev. Mol. Cell Biol. 8, 195-208. doi: 10.1038/nrm2114

Subbarao, E. K., London, W., and Murphy, B. R. (1993). A single amino acid in the PB2 gene of influenza A virus is a determinant of host range. $J$. Virol. 67, 1761-1764. doi: 10.1128/jvi.67.4.1761-1764.1993

Sun, Y., Hu, Z., Zhang, X., Chen, M., Wang, Z., Xu, G., et al. (2020). An $\mathrm{R} 195 \mathrm{~K}$ mutation in the PA-X protein increases the virulence and transmission of influenza A virus in mammalian hosts. J. Virol. 94:e01817-19. doi: 10.1128/ JVI.01817-19

Sun, Y., Qin, K., Wang, J., Pu, J., Tang, Q., Hu, Y., et al. (2011). High genetic compatibility and increased pathogenicity of reassortants derived from avian 
H9N2 and pandemic H1N1/2009 influenza viruses. Proc. Natl. Acad. Sci. U. S. A. 108, 4164-4169. doi: 10.1073/pnas.1019109108

Tarendeau, F., Boudet, J., Guilligay, D., Mas, P. J., Bougault, C. M., Boulo, S., et al. (2007). Structure and nuclear import function of the C-terminal domain of influenza virus polymerase PB2 subunit. Nat. Struct. Mol. Biol. 14, 229-233. doi: $10.1038 / \mathrm{nsmb} 1212$

Thiele, S., Stanelle-Bertram, S., Beck, S., Kouassi, N. M., Zickler, M., Müller, M., et al. (2020). Cellular importin- $\alpha 3$ expression dynamics in the lung regulate antiviral response pathways against influenza A virus infection. Cell Rep. 31:107549. doi: 10.1016/j.celrep.2020.107549

Tong, S., Zhu, X., Li, Y., Shi, M., Zhang, J., Bourgeois, M., et al. (2013). New world bats harbor diverse influenza A viruses. PLoS Pathog. 9:e1003657. doi: 10.1371/journal.ppat.1003657

Vasin, A. V., Temkina, O. A., Egorov, V. V., Klotchenko, S. A., Plotnikova, M. A., and Kiselev, O. I. (2014). Molecular mechanisms enhancing the proteome of influenza A viruses: an overview of recently discovered proteins. Virus Res. 185, 53-63. doi: 10.1016/j.virusres.2014.03.015

Wang, C., Lee, H. H., Yang, Z. F., Mok, C. K., and Zhang, Z. (2016). PB2Q591K mutation determines the pathogenicity of avian H9N2 influenza viruses for mammalian species. PLoS One 11:e0162163. doi: 10.1371/journal. pone. 0169417

Wong, S. S., and Yuen, K. Y. (2006). Avian influenza virus infections in humans. Chest 129, 156-168. doi: 10.1378/chest.129.1.156

Xu, R., de Vries, R. P., Zhu, X. Y., Nycholat, C. M., McBride, R., Yu, W. L., et al. (2013). Preferential recognition of avian-like receptors in human influenza A H7N9 viruses. Science 342, 1230-1235. doi: 10.1126/ science. 1243761

Xu, G., Zhang, X., Liu, Q., Bing, G., Hu, Z., Sun, H., et al. (2017). PA-X protein contributes to virulence of triple-reassortant H1N2 influenza virus by suppressing early immune responses in swine. Virology 508, 45-53. doi: 10.1016/j.virol.2017.05.002

Xu, G., Zhang, X., Sun, Y., Liu, Q., Sun, H., Xiong, X., et al. (2016). Truncation of C-terminal 20 amino acids in PA-X contributes to adaptation of swine influenza virus in pigs. Sci. Rep. 6:21845. doi: 10.1038/srep21845

Xue, R., Tian, Y., Hou, T., Bao, D., Chen, H., Teng, Q., et al. (2018). H9N2 influenza virus isolated from minks has enhanced virulence in mice. Transbound. Emerg. Dis. 65, 904-910. doi: 10.1111/tbed.12805

Yang, Z. F., Mok, C. K., Peiris, J. S., and Zhong, N. S. (2015). Human infection with a novel avian influenza A (H5N6) virus. N. Engl. J. Med. 373, 487-489. doi: 10.1056/NEJMc1502983

Yin, C., Khan, J. A., Swapna, G. V., Ertekin, A., Krug, R. M., Tong, L., et al. (2007). Conserved surface features form the double-stranded RNA binding site of non-structural protein 1 (NS1) from influenza A and B viruses. J. Biol. Chem. 282, 20584-20592. doi: 10.1074/jbc.M611619200

Yuan, P. W., Bartlam, M., Lou, Z. Y., Chen, S. D., Zhou, J., He, X. J., et al. (2009). Crystal structure of an avian influenza polymerase PA(N) reveals an endonuclease active site. Nature 458, 909-912. doi: 10.1038/nature07720

Zhang, H., Li, H., Wang, W., Wang, Y., Han, G. Z., Chen, H., et al. (2020a). A unique feature of swine ANP32A provides susceptibility to avian influenza virus infection in pigs. PLoS Pathog. 16:e1008330. doi: 10.1371/journal. ppat.1008330

Zhang, Y., Zhang, Q., Kong, H., Jiang, Y., Gao, Y., Deng, G., et al. (2013). H5N1 hybrid viruses bearing 2009/H1N1 virus genes transmit in Guinea pigs by respiratory droplet. Science 340, 1459-1463. doi: 10.1126/science.1229455

Zhang, H., Zhang, Z., Wang, Y., Wang, M., Wang, X., Zhang, X., et al. (2019). Fundamental contribution and host range determination of ANP32A and ANP32B in influenza A virus polymerase activity. J. Virol. 93:e00174-19. doi: 10.1128/JVI.00174-19

Zhang, Z., Zhang, H., Xu, L., Guo, X., Wang, W., Ji, Y., et al. (2020b). Selective usage of ANP32 proteins by influenza B virus polymerase: implications in determination of host range. PLoS Pathog. 16:e1008989. doi: 10.1371/journal. ppat.1008989

Zielecki, F., Semmler, I., Kalthoff, D., Voss, D., Mauel, S., Gruber, A. D., et al. (2010). Virulence determinants of avian H5N1 influenza A virus in mammalian and avian hosts: role of the C-terminal ESEV motif in the viral NS1 protein. J. Virol. 84, 10708-10718. doi: 10.1128/JVI.00610-10

Conflict of Interest: The authors declare that the research was conducted in the absence of any commercial or financial relationships that could be construed as a potential conflict of interest.

Publisher's Note: All claims expressed in this article are solely those of the authors and do not necessarily represent those of their affiliated organizations, or those of the publisher, the editors and the reviewers. Any product that may be evaluated in this article, or claim that may be made by its manufacturer, is not guaranteed or endorsed by the publisher.

Copyright (c) 2021 Zhang, Liu, Bai, Zhao, Li, Xu and Zhang. This is an open-access article distributed under the terms of the Creative Commons Attribution License (CC BY). The use, distribution or reproduction in other forums is permitted, provided the original author(s) and the copyright owner(s) are credited and that the original publication in this journal is cited, in accordance with accepted academic practice. No use, distribution or reproduction is permitted which does not comply with these terms. 